\title{
Attractors for the 3D autonomous and nonautonomous Brinkman-Forchheimer equations
}

\author{
Lingrui Zhang ${ }^{1 *}$, Keqin Su ${ }^{2}$ and Shenglan Wen ${ }^{3}$
}

\section{"Correspondence:}

zhanglingrui@126.com

${ }^{1}$ College of Education and Teacher

Development, Henan Normal

University, Xinxiang, 453007,

P.R. China

Full list of author information is

available at the end of the article

\begin{abstract}
We consider the large-time behavior such as the existence of attractors for the 3D autonomous and nonautonomous Brinkman-Forchheimer equations. By means of the decomposition method we overcome the difficulties for the existence of absorbing sets and asymptotical compactness of the semigroup generated by a global solution to prove the attractors for the autonomous Brinkman-Forchheimer equation. Under suitable assumptions on the external force $\sigma(t, x)$ and initial data $u_{\tau}(x)$, we prove the existence of a uniform attractor for a 3D nonautonomous Brinkman-Forchheimer equation. Moreover, we apply the theory of weak continuity and weak convergence method to establish the asymptotical compactness of the processes.
\end{abstract}

MSC: 35B40; 35Q99; 80A22

Keywords: Brinkman-Forchheimer equation; symbol; processes; uniform attractor

\section{Introduction}

The large-time behavior of global solutions and the associated infinite dimensional dynamical systems have become the essential aspects in the field of nonlinear evolutionary equations such as the existence of global attractors, inertial manifold, uniform attractors, pullback attractors, and their fractal dimensions for the autonomous and nonautonomous systems with the unique solution have attracted attention of many mathematicians since the 1980s.

From the physical viewpoint, Gilver and Altobelli [1] obtained a determination of effective viscosity for the Brinkman-Forchheimer flow model. Nield [2] dealt with an important and classical problem (the momentum equation in a porous medium, i.e., the background of the Brinkman-Forchheimer equation) involving the fluid mechanics of the interface region between a porous medium and a fluid layer. Vafai and Kim [3, 4] obtained an exact solution to this problem using a Brinkman-Forchheimer-extended Darcy equation (generalized momentum equation). Whitaker [5] investigated the theoretical development of the Forchheimer equation.

In mathematical analysis, for the autonomous Brinkman-Forchheimer equation, using condition-(C) method, Uğurlu [6] and Ouyang and Yan [7] showed the existence of global attractor in $H_{0}^{1}(\Omega)$. Çelebi et al. [8] proved the continuous dependence of solutions of the

(c) 2016 Zhang et al. This article is distributed under the terms of the Creative Commons Attribution 4.0 International License (http://creativecommons.org/licenses/by/4.0/), which permits unrestricted use, distribution, and reproduction in any medium, provided you give appropriate credit to the original author(s) and the source, provide a link to the Creative Commons license, and indicate if changes were made. 
Brinkman-Forchheimer equations on the Brinkman and Forchheimer coefficients $v$ and $\gamma$ in $H^{1}$-norm, which reflects the effect of small changes in coefficients of equations on the solutions. It was shown in $[9,10]$, and [11] that the Brinkman-Forchheimer equation has a global solution, which continuously depends on the coefficients. Moreover, they also derived the convergence of corresponding solutions as coefficients tend to zero. In [6] and [12], the existence of global attractors of the Brinkman-Forchheimer equation is obtained by different methods. However, there are fewer results for the nonautonomous case. Recently, in [13], the existence of $\mathcal{D}$-pullback attractors for 3D nonautonomous BrinkmanForchheimer equation is deduced by establishing the $\mathcal{D}$-pullback asymptotical compactness of $\theta$-cocycle. More references about the BF equation and relate models, please refer to $[2,14-17]$.

In this paper, we consider the large time behavior (i.e., the existence of global and uniform attractors for the autonomous and nonautonomous Brinkman-Forchheimer equations by decomposition method and weak continuous method to establish asymptotical compactness for the semigroups and processes, respectively) for the 3D BrinkmanForchheimer equation that governs the motion of fluid in a saturated porous medium:

$$
\left\{\begin{array}{l}
u_{t}-v \Delta u+\alpha u+\beta|u| u+\gamma|u|^{2} u+\nabla p=\sigma(t, x), \quad(x, t) \in \Omega \times \mathbb{R}^{+}, \\
\operatorname{div} u=0, \quad(x, t) \in \Omega \times \mathbb{R}^{+}, \\
\left.u(t, x)\right|_{\partial \Omega}=0, \quad t \in \mathbb{R}^{+} \\
u(\tau, x)=u_{\tau}(x), \quad x \in \Omega, \tau \geq 0 .
\end{array}\right.
$$

Here $\Omega \subset \mathbb{R}^{3}$ is a bounded domain with sufficiently smooth boundary $\partial \Omega, u=u(t, x)=$ $\left(u_{1}(t, x), u_{2}(t, x), u_{3}(t, x)\right)$ is the velocity vector field, $p$ is the pressure, $v>0$ is the Brinkman kinematic viscosity coefficient, $\alpha>0$ is the Darcy coefficient, and $\beta>0$ and $\gamma>0$ are the Forchheimer coefficients.

In our paper, we use the composition to prove the existence of attractor for an autonomous system by weak continuous method, which is introduced by Ball to derive the uniform attractors for a nonautonomous system; this is different from the papers [18] and [19].

The paper is organized as follows. In Section 2, we establish the existence of global solutions for the 3D Brinkman-Forchheimer equation and introduce some useful preliminaries and notation. Some concepts and theorems on the theory of global attractors for autonomous dynamical systems are stated in Section 3. Some concepts and theorems on the theory of uniform attractors for nonautonomous dynamical systems are arranged in Section 4. Finally, the existence of uniform attractors for the 3D nonautonomous BrinkmanForchheimer equation is proved.

\section{Global existence of solutions for 3D Brinkman-Forchheimer equations}

Throughout this paper, $C$ will stand for a generic positive constant, depending on $\Omega$ and some constants, but independent of the choice of the initial time $\tau \in \mathbb{R}$ and $t$.

The Hausdorff semidistance in $X$ from one set $B_{1}$ to another set $B_{2}$ is defined as

$$
\operatorname{dist}_{X}\left(B_{1}, B_{2}\right)=\sup _{b_{1} \in B_{1}} \inf _{b_{2} \in B_{2}}\left\|b_{1}-b_{2}\right\|_{X}
$$

We denote by $L^{p}(\Omega)$ and $H^{m}(\Omega)$ the general Lebesgue and Sobolev spaces, respectively. We set $E:=\left\{u \mid u \in\left(C_{0}^{\infty}(\Omega)\right)^{3}, \operatorname{div} u=0\right\}, H$ is the closure of the set $E$ in $\left(L^{2}(\Omega)\right)^{3}$ topology, 
and $V$ is the closure of the set $E$ in $\left(H_{0}^{1}(\Omega)\right)^{3}$ topology, that is, we set $V=H \cap\left(H_{0}^{1}(\Omega)\right)^{3}$. We set $\mathcal{E}:=\left\{u \mid u \in\left(C_{0}^{\infty}(\Omega)\right)^{3}, \operatorname{div} u=0\right\}, H$ is the closure of $\mathcal{E}$ in $\left(L^{2}(\Omega)\right)^{3}$ topology, $V$ is the closure of $\mathcal{E}$ in $\left(H^{1}(\Omega)\right)^{3}$ topology, $W$ is the closure of $\mathcal{E}$ in $\left(H^{2}(\Omega)\right)^{3}$ topology, and $H^{\prime}$ and $V^{\prime}$ are dual spaces of $H$ and $V$, respectively. Clearly, $V \hookrightarrow H \equiv H^{\prime} \hookrightarrow V^{\prime}$, where the injection is dense and continuous. By $\|\cdot\|$ and $(\cdot, \cdot)$ we denote the norm and inner product of $H$, that is,

$$
(u, v)=\sum_{j=1}^{3} \int_{\Omega} u_{j}(x) v_{j}(x) d x, \quad\|u\|^{2}=(u, u), u, v \in\left(L^{2}(\Omega)\right)^{3}
$$

and $\|\cdot\|_{V}$ and $((\cdot, \cdot))$ denote the norm and inner product in $V$, that is,

$$
((u, v))=\sum_{i, j=1}^{3} \int_{\Omega} \frac{\partial u_{j}}{\partial x_{i}} \cdot \frac{\partial v_{j}}{\partial x_{i}} d x, \quad u, v \in V
$$

and

$$
\|u\|_{V}^{2}:=\sum_{i=1, j=1}^{3}\left\|\partial_{i} u_{j}\right\|_{L^{2}(\Omega)}^{2}, \quad u \in V
$$

Let $P$ be the Helmholtz-Leray orthogonal projection operator from $\left(L^{2}(\Omega)\right)^{3}$ onto $H$. Let $A u=-P \Delta u$ be the Stokes operator with domain $D(A)=\left(H^{2}(\Omega)\right)^{3} \cap\left(H_{0}^{1}(\Omega)\right)^{3}$, and let $\lambda$ be the first eigenvalue of $A$; then the operator $A: V \rightarrow V^{\prime}$ has the property $\langle A u, v\rangle=((u, v))$ for all $u, v \in V$.

The family of functions in $L_{\text {loc }}^{2}(\mathbb{R} ; H)$ denotes a local Bochner integration function class, and $L_{b}^{2}(\mathbb{R} ; H)$ denotes all translation bounded functions $\sigma(x, t)$ that satisfy $\sup _{t \in \mathbb{R}} \int_{t}^{t+1} \| \sigma(s$, $x) \|_{H}^{2} d s<+\infty$ for all $\sigma \in L_{\text {loc }}^{2}(\mathbb{R} ; H)$, that is, $\sigma$ is translation bounded in $L_{\text {loc }}^{2}(\mathbb{R} ; H)$. Obviously, $L_{b}^{2}(\mathbb{R} ; H) \subset L_{\text {loc }}^{2}(\mathbb{R} ; H)$.

Problem (1.1) can be written in an abstract form

$$
\begin{aligned}
& u_{t}+v A u+\alpha u+B(u)=\sigma(t, x), \\
& \operatorname{div} u=0, \\
& \left.u\right|_{\partial \Omega}=0, \\
& u(\tau, x)=u_{\tau},
\end{aligned}
$$

where the pressure $p$ has disappeared by application of the Leray-Helmholtz projection $P$, $B(u)=P F(u), F(u)=\beta|u| u+\gamma|u|^{2} u$. Clearly, system (2.5)-(2.8) is equivalent to (1.1).

Similarly to the autonomous case, the existence and uniqueness of a global solution for (1.1) can be derived by a standard method as in $[8,12]$, or [10].

Theorem 2.1 Assume that $\sigma \in L_{\mathrm{loc}}^{2}(\mathbb{R}, H)$ and $u_{\tau} \in H$. Then problem (2.5)-(2.8) possesses a unique global solution $u(t, x)$, which satisfies

$$
u \in C([\tau,+\infty) ; H) \cap L^{2}(\tau, T ; V) \cap L^{4}\left(\tau, T ;\left(L^{4}(\Omega)\right)^{3}\right) .
$$


Moreover, choosing a nonautonomous fixed external force $\sigma_{0}(t, x) \in L_{b}^{2}(\mathbb{R}, H)$, the global solution $u(t, x)$ generates a process $\left\{U_{\sigma}(\tau, t)\right\}(\tau \in \mathbb{R}, t>\tau, \sigma \in \Sigma)$, which is continuous with respect to $u_{\tau}$, where $\sigma$ is a symbol that belongs to the symbol space $\Sigma=\mathcal{H}\left(\sigma_{0}\right)=\left[\left\{\sigma_{0}(s+\right.\right.$ h) $\mid h \in \mathbb{R}\}]_{L_{\text {loc }}^{2}(\mathbb{R}, H)}$ with $[\cdot]_{E}$ meaning the closure in the topology of $E$.

Remark 2.1 For the autonomous system, Theorem 2.1 also holds if the external force satisfies $\sigma(x) \in H$.

\section{Existence of global attractors for autonomous system}

The autonomous system can be written in the abstract form

$$
\begin{aligned}
& u_{t}+v A u+\alpha u+\beta|u| u+\gamma|u|^{2} u=h(x), \quad x \in \Omega, \\
& \operatorname{div} u=0,
\end{aligned}
$$

with the slip condition and initial data.

Theorem 3.1 For any $h \in V^{\prime}$ and $u_{\tau} \in H$, the semigroup $\left\{S(t), t \geq \tau \in \mathbb{R}^{+}\right\}$generated by system (3.1)-(3.2) has a global attractor $\mathcal{A}$, which is invariant, compact, and attracts all bounded subsets of $H$ in the topology of $H$.

The proof can be divided into two steps. First, we prove the existence of absorbing sets; next, we shall use the decomposition method to prove the asymptotical smoothness for the semigroup.

Since the injection $H \hookrightarrow V^{\prime}$ is dense, for any $h \in V^{\prime}$, we can find a function $h^{\varepsilon} \in H$ depending on $h$ and $\varepsilon$ such that

$$
\left\|h-h^{\varepsilon}\right\| \leq \varepsilon
$$

Using a technique similar to that in Zelik [20], we decompose the semigroup into $S(t) u_{0}=S_{v}(t) u_{0}+S_{w}(t) u_{0}$ with $S_{v}(t) u_{0}=v(t)$ and $S_{w}(t) u_{0}=w(t)$ that satisfy the following linear and nonlinear equations respectively:

$$
\left\{\begin{array}{l}
v_{t}+v A v+\alpha v=h-h^{\varepsilon} \\
\operatorname{div} v=0 \\
v(x, 0)=u_{\tau}(x)
\end{array}\right.
$$

and

$$
\left\{\begin{array}{l}
w_{t}+v A w+\alpha w+\beta|u| u+\gamma|u|^{2} u=h^{\varepsilon} \\
\operatorname{div} w=0, \\
w(x, 0)=0, \quad x \in \Omega
\end{array}\right.
$$

Lemma 3.1 Let the external force $h \in V^{\prime}$, and let $u_{\tau} \in H$. Then the semigroup has a bounded absorbing set $B_{0}$ in $H$, where

$$
B_{0}=\left\{u \in H:\|u\|_{H} \leq \rho\right\}
$$

is a bounded set in $H$. 
Proof Taking inner product of (3.1) with $u$ and integrating by parts over $\Omega$, we have

$$
\begin{aligned}
\frac{1}{2} \frac{d}{d t}\|u\|^{2}+v\|\nabla u\|^{2}+\alpha\|u\|^{2}+\beta\|u\|_{L^{3}}^{3}+\gamma\|u\|_{L^{4}}^{4} & =\langle h(x), u\rangle \\
& \leq \lambda v\|u\|^{2}+\frac{\|h\|_{V^{\prime}}^{2}}{\lambda v},
\end{aligned}
$$

where $\|\cdot\|$ denotes the $H$ norm, and $\|\nabla(\cdot)\|$ is equivalent to the $V$ norm.

The Poincaré inequality $\|\nabla u\|^{2} \geq \lambda\|u\|^{2}$ implies

$$
\frac{1}{2} \frac{d}{d t}\|u\|^{2}+\lambda v\|u\|^{2}+\alpha\|u\|^{2} \leq \frac{1}{\lambda v}\|h(x)\|_{V^{\prime}}^{2}+\lambda v\|u\|^{2},
$$

that is,

$$
\frac{d}{d t}\|u\|^{2}+2 \alpha\|u\|^{2} \leq \frac{2}{\lambda v}\|h(x)\|_{V^{\prime}}^{2}
$$

By the Gronwall inequality we have

$$
\|u(x, t)\|^{2} \leq e^{-2 \alpha t}\left\|u_{\tau}\right\|^{2}+\frac{1}{\alpha \nu \lambda}\|h(x)\|_{V^{\prime}}^{2} .
$$

Choosing $\rho^{2}=e^{-2 \alpha t}\left\|u_{\tau}\right\|^{2}+\frac{1}{\alpha \nu \lambda}\|h(x)\|_{V^{\prime}}^{2}$, we get that $B_{0}=\left\{u:\|u\|_{H}^{2} \leq \rho^{2}\right\}$ is an absorbing ball for the semigroup $\{S(t)\}_{t \geq 0}$ in $H$.

Theorem 3.2 For any $h \in V^{\prime}$, the semigroup $\{S(t) \mid t \geq 0\}$ generated by system (3.1)-(3.2) with the initial data and boundary value problem in (1.1) is asymptotically smooth in $H$.

Proof We shall use decomposition method to achieve the result in the following lemmas.

Lemma 3.2 For system (3.4), there exists a constant $\varepsilon=\varepsilon(h, \delta)$ such that the solution of (3.4) satisfies, for any $t \geq 0$,

$$
\left\|S_{v}(t) u_{\tau}\right\|_{H}^{2}=\|v(x, t)\|_{H}^{2} \leq Q\left(\left\|u_{\tau}\right\|_{H}\right) e^{-2 \alpha t}+\delta
$$

where $Q$ is a positive increasing function on $[0,+\infty)$.

Proof Multiplying (3.4) with $v(t)$ and integrating over $\Omega$, we deduce

$$
\frac{1}{2} \frac{d}{d t}\|v\|^{2}+v\|\nabla v\|^{2}+\alpha\|v\|^{2} \leq \frac{1}{\nu \lambda}\left\|h-h^{\varepsilon}\right\|_{V^{\prime}}^{2}+v \lambda\|v\|^{2},
$$

which means that

$$
\frac{d}{d t}\|v\|^{2}+2 \alpha\|v\|^{2} \leq \frac{2}{v \lambda}\left\|h-h^{\varepsilon}\right\|_{V^{\prime}}^{2} .
$$

Applying the Gronwall lemma, we have

$$
\|v\|_{H}^{2} \leq Q\left(\left\|u_{\tau}\right\|_{H}\right) e^{-2 \alpha t}+\frac{1}{\alpha \nu \lambda}\left\|h-h^{\varepsilon}\right\|_{V^{\prime}}^{2}
$$

Choosing $\varepsilon \leq v \alpha \lambda \delta$, we complete the proof. 
Lemma 3.3 For any time $T>0$ and any $\varepsilon>0$, there exists a nonnegative constant $M>$ 0 depending on the constants $T, h^{\varepsilon},\left\|u_{\tau}\right\|_{H}$ such that the solution to system (3.5) has the property

$$
\left\|S_{w}(t) u_{\tau}\right\|_{H}^{2}=\|w(t)\|_{H}^{2} \leq M
$$

Proof Taking inner product of (3.5) with $w(t)$, we obtain

$$
\frac{1}{2} \frac{d}{d t} \int_{\Omega}|w|^{2} d x+v \int_{\Omega}|\nabla w|^{2} d x+\alpha \int_{\Omega}|w|^{2} d x+(B(u), w)=\left\langle h^{\varepsilon}, w\right\rangle,
$$

where $B(u)$ is defined in Section 2.

By the Cauchy inequality and the Sobolev compact embedding $V \hookrightarrow L^{6}(\Omega) \hookrightarrow L^{4}(\Omega)$ we have

$$
\left\langle h^{\varepsilon}, w\right\rangle \leq C\left\|h^{\varepsilon}\right\|\|w\| \leq \frac{2}{3 \alpha}\left\|h^{\varepsilon}\right\|^{2}+\frac{3 \alpha}{2} \int_{\Omega}|w|^{2} d x
$$

and

$$
\begin{aligned}
|(B(u), w)| & \leq C\left(\|u\|_{L^{4}}^{4}+\|w\|+\|u\|_{L^{4}}^{4}+\left\|u^{2} w^{2}\right\|\right) \\
& \leq C\|u\|_{L^{4}}^{4}+\frac{C}{\varepsilon}\|u\|_{L^{4}}^{4}+C \varepsilon\|w\|_{L^{4}}^{4} \\
& \leq C\left(\|u\|_{L^{4}}^{4}+\|v\|_{L^{4}}^{4}\right) \\
& \leq C\left(\|u\|_{L^{4}}^{4}+1\right) .
\end{aligned}
$$

Substituting (3.16) and (3.17) into (3.15) and then using Theorem 2.1 and Lemma 3.2, we derive

$$
\frac{d}{d t} \int_{\Omega}|w|^{2} d x \leq C^{\prime}\left(\int_{\Omega}|w|^{2} d x+1\right)
$$

where the constant $C^{\prime}=C^{\prime}\left(v, \alpha, \beta, \gamma, \lambda,\left\|h^{\varepsilon}\right\|^{2},\left\|u_{\tau}\right\|^{2}\right)>0$, which yields

$$
\int_{\Omega}|w|^{2} d x \leq e^{C^{\prime} t}, \quad t \geq 0
$$

Since the embedding $V \hookrightarrow H$ is compact, we can deduce the asymptotical smoothness for the semigroup, which completes the proof of the lemma and Theorem 3.1.

\section{Some preliminaries of uniform attractors}

For the nonautonomous evolutionary system

$$
\partial_{t} u=A_{\sigma(t)} u,
$$

where $A_{\sigma(t)}$ is an operator, we introduce the two-parameter processes $U_{\sigma}(t, \tau): E \rightarrow E$ that describe the solution trajectory, where $U_{\sigma}(t, \tau): E \rightarrow E$ satisfies

$$
U_{\sigma}(t, s) U_{\sigma}(s, \tau)=U_{\sigma}(t, \tau), \quad t \geq s \geq \tau, \tau \in \mathbb{R},
$$




$$
U_{\sigma}(\tau, \tau)=I \quad \text { (identity operator) }
$$

The translation semigroup $\{S(h): \mathbb{R} \rightarrow \mathbb{R} \mid h \geq 0\}$ maps it into itself and satisfies

$$
\begin{aligned}
& \sigma_{0}(s+h)=S(h) \sigma_{0}(s), \\
& U_{\sigma}(t+h, \tau+h)=U_{S(h) \sigma}(t, \tau),
\end{aligned}
$$

for any $\sigma \in \Sigma, t \geq \tau, \tau \in \mathbb{R}, h \geq 0$, where we choose arbitrary but fixed $\sigma_{0} \in L_{b}^{2}(\mathbb{R} ; W)$, $\Sigma=\mathcal{H}\left(\sigma_{0}\right)=\left[\left\{\sigma_{0}(\cdot+h) \mid h \in \mathbb{R}\right\}\right]_{L_{\text {loc }}^{2}(\mathbb{R} ; W)}$ is the symbol space, and the extended phase space of the nonautonomous system (3.1) is $E \times \mathcal{H}\left(\sigma_{0}\right)$.

Definition 4.1 Denote by $\mathcal{B}(E)$ all bounded (in the norm of $E$ ) sets in $E$. Then a set $\mathcal{B}_{0}$ is said to be a uniformly (w.r.t. $\left.\sigma \in \mathcal{H}\left(\sigma_{0}\right)\right)$ absorbing for the family of processes $\left\{U_{\sigma}(t, \tau)\right\}$, $\sigma \in \mathcal{H}\left(\sigma_{0}\right)$ if for any set $\mathcal{B} \in \mathcal{B}(E)$, there exists some time $t_{0}=t_{0}(\mathcal{B}, \tau) \geq \tau$ such that $\bigcup_{\sigma \in \mathcal{H}\left(\sigma_{0}\right)} U_{\sigma}(t, \tau) \mathcal{B} \subseteq \mathcal{B}_{0}$ for all $t \geq t_{0}$.

Definition 4.2 A closed set $\Lambda \subset E$ is called a uniform (w.r.t. $\sigma \in \mathcal{H}\left(\sigma_{0}\right)$ ) attractor of the processes $\left\{U_{\sigma}(t, \tau)\right\}, \sigma \in \mathcal{H}\left(\sigma_{0}\right)$ if for all $\mathcal{B} \in \mathcal{B}(E)$ and arbitrary fixed $\tau \in \mathbb{R}$, there exists some time $t_{0}=t_{0}(\mathcal{B}, \tau) \geq \tau$ such that

$$
\lim _{t \rightarrow+\infty} \sup _{\sigma \in \mathcal{H}\left(\sigma_{0}\right)} \operatorname{dist}_{E}\left(U_{\sigma}(t, \tau) \mathcal{B}, \Lambda\right)=0
$$

for all $t>t_{0}$, where $\operatorname{dist}_{E}(X, Y)$ denotes the Hausdorff semidistance between sets $X$ and $Y$ in the topology space $E$.

Definition 4.3 The family of processes $\left\{U_{\sigma}(t, \tau)\right\}, \sigma \in \mathcal{H}\left(\sigma_{0}\right)$ is said to be $(E \times \Sigma, E)$ continuous if the map $(u, \sigma) \mapsto U_{\sigma}(t, \tau)$ is continuous from $E \times \Sigma$ to $E$ for all fixed $t \geq \tau$, $\tau \in \mathbb{R}, \sigma \in \Sigma$.

Definition 4.4 The family of processes $\left\{U_{\sigma}(t, \tau)\right\}, \sigma \in \mathcal{H}\left(\sigma_{0}\right)$ is said to be uniformly (w.r.t. $\left.\sigma \in \mathcal{H}\left(\sigma_{0}\right)\right)$ asymptotically compact in $E$ if $\left\{U_{\sigma^{n}}\left(t_{n}, \tau\right) u_{\tau}^{(n)}\right\}$ is precompact in $E$ whenever $\left\{u_{\tau}^{(n)}\right\}$ is bounded in $E, \sigma^{(n)} \subset \mathcal{H}\left(\sigma_{0}\right)$, and $\left\{t_{n}\right\} \subset \mathbb{R}_{\tau}, t_{n} \rightarrow+\infty$ as $n \rightarrow+\infty$.

Definition 4.5 A closed set $\mathcal{A} \subset E$ is called a uniform (w.r.t., $\sigma \in \mathcal{H}\left(\sigma_{0}\right)$ ) global attractor of the processes $\left\{U_{\sigma}(t, \tau)\right\}, \sigma \in \mathcal{H}\left(\sigma_{0}\right)$, acting on $E$ if $\mathcal{A}$ is a closed and uniformly attracting set in $E$ and $\mathcal{A}$ satisfies the following minimality property: $\mathcal{A}$ belongs to any closed uniformly attracting set of the processes $\left\{U_{\sigma}(t, \tau) \mid \sigma \in \mathcal{H}\left(\sigma_{0}\right)\right\}$.

Theorem 4.1 The $(E \times \Sigma, E)$-continuous processes $\left\{U_{\sigma}(t, \tau)\right\}, \sigma \in \mathcal{H}\left(\sigma_{0}\right)$, have a uniform (w.r.t. $\sigma \in \Sigma$ ) compact attractor $\mathcal{A}_{\Sigma}$ in $E$ that satisfies

$$
\mathcal{A}_{\Sigma}=\omega_{0, \Sigma}\left(B_{0}\right)=\omega_{\tau, \Sigma}\left(B_{0}\right)=\bigcup_{B \in \mathcal{B}(E)} \omega_{\tau, \Sigma}(B)
$$

if

(i) there exists a bounded uniform (w.r.t. $\sigma \in \Sigma$ ) absorbing set $B_{0}$ of processes $\left\{U_{\sigma}(t, \tau)\right\}$, $\sigma \in \Sigma$, and

(ii) the processes $\left\{U_{\sigma}(t, \tau)\right\}(\sigma \in \Sigma)$ are uniformly asymptotically compact. 
Proof See, for example, [21].

\section{Uniform attractors for 3D nonautonomous Brinkman-Forchheimer equations} in $H$

Choose an arbitrary nonautonomous force but fixed $\sigma_{0}(x, t) \in L_{b}^{2}(\mathbb{R}, H)$, that is,

$$
\sup _{t \in \mathbb{R}} \int_{t}^{t+1}\left\|\sigma_{0}(s)\right\|_{H}^{2} d s<+\infty
$$

Taking $\Sigma=\mathcal{H}\left(\sigma_{0}\right)$ (defined in Theorem 2.1) as the symbol space of problem (2.5)-(2.8), $\forall \sigma \in \Sigma$ is called the symbol of system (2.5)-(2.8).

Obviously, $\mathcal{H}\left(\sigma_{0}\right)$ is strictly invariant under the acting of the translation semigroup $\{S(h)\}_{h \geq 0}$, that is, $S(h) \mathcal{H}\left(\sigma_{0}\right) \equiv \mathcal{H}\left(\sigma_{0}\right)$ for all $h \geq 0$.

By Theorem 2.1 the global solution generates the process class $\left\{U_{\sigma}(t, \tau), t \geq \tau, \tau \in \mathbb{R}\right\}$, $\sigma \in \mathcal{H}\left(\sigma_{0}\right)$, that is, $U_{\sigma}(t, \tau) u_{\tau}=u(t)$, where $u(t)$ is the solution of problem (2.5)-(2.8) with symbol $\sigma \in \Sigma$ and initial data $u_{\tau} \in H$.

Lemma 5.1 Let the external force $\sigma \in \Sigma$ and $u_{\tau} \in H$. Then the process has a bounded uniform (w.r.t. $\left.\sigma \in \mathcal{H}\left(\sigma_{0}\right)\right)$ absorbing set $B_{0}$ in $H$, where

$$
B_{0}=\left\{u \in H:\|u\|_{H} \leq C\left\|\sigma_{0}\right\|_{L_{b}^{2}(\mathbb{R} ; H)} \doteq \rho\right\}
$$

is a bounded set in $H$.

Proof Multiplying (1.1) with $u$ and integrating on $\Omega$, by the Young inequality we conclude

$$
\begin{gathered}
\frac{1}{2} \frac{d}{d t}\|u\|^{2}+v\|\nabla u\|^{2}+\alpha\|u\|^{2}+\beta\|u\|_{L^{3}}^{3}+\gamma\|u\|_{L^{4}}^{4} \\
\quad=\int_{\Omega} \sigma(t, x) u d x \leq \frac{\alpha}{2}\|u\|^{2}+\frac{2\|\sigma\|^{2}}{\alpha} .
\end{gathered}
$$

Then integrating over $[\tau, t]$, we have

$$
\begin{aligned}
& \|u\|^{2}+\int_{\tau}^{t}\left(2 v\|\nabla u\|^{2}+\alpha\|u\|^{2}+2 \beta\|u\|_{L^{3}}^{3}+2 \gamma\|u\|_{L^{4}}^{4}\right) d s \\
& \leq \frac{4}{\alpha} \int_{\tau}^{t}\|\sigma(s)\|^{2} d s+\left\|u_{\tau}\right\|^{2} .
\end{aligned}
$$

Hence,

$$
\|u\|^{2}+\int_{\tau}^{t}\left(2 \lambda_{1} v+\alpha\right)\|u\|^{2} d s \leq \frac{4}{\alpha} \int_{\tau}^{t}\|\sigma(s)\|^{2} d s+\left\|u_{\tau}\right\|^{2},
$$

where $\lambda_{1}$ is the first eigenvalue in the Poincaré inequality.

By Gronwall's inequality we derive

$$
\begin{aligned}
\|u(t)\|^{2} & \leq\left\|u_{\tau}\right\|^{2} e^{-\left(2 \lambda_{1} v+\alpha\right)(t-\tau)}+\frac{4}{\alpha} \int_{\tau}^{t} e^{\left(2 \lambda_{1} v+\alpha\right)(s-t)}\|\sigma(s)\|^{2} d s \\
& \leq\left\|u_{\tau}\right\|^{2} e^{-\left(2 \lambda_{1} v+\alpha\right)(t-\tau)}+\frac{4}{\alpha}\left(\int_{t-1}^{t} e^{\left(2 \lambda_{1} v+\alpha\right)(s-t)}\|\sigma(s)\|^{2} d s\right.
\end{aligned}
$$




$$
\begin{aligned}
& \left.+\int_{t-2}^{t-1} e^{\left(2 \lambda_{1} v+\alpha\right)(s-t)}\|\sigma(s)\|^{2} d s+\cdots\right) \\
\leq & \left\|u_{\tau}\right\|^{2} e^{-\left(2 \lambda_{1} v+\alpha\right)(t-\tau)}+\frac{4}{\alpha}\left(\int_{t-1}^{t}\|\sigma(s)\|^{2} d s\right. \\
& \left.+e^{-\left(2 \lambda_{1} v+\alpha\right)} \int_{t-2}^{t-1}\|\sigma(s)\|^{2} d s+e^{-2\left(2 \lambda_{1} v+\alpha\right)} \int_{t-3}^{t-2}\|\sigma(s)\|^{2} d s+\cdots\right) \\
\leq & \left\|u_{\tau}\right\|^{2} e^{-\left(2 \lambda_{1} v+\alpha\right)(t-\tau)}+\frac{4}{\alpha}\left(1+e^{-\left(2 \lambda_{1} v+\alpha\right)}+e^{-2\left(2 \lambda_{1} v+\alpha\right)}+\cdots\right)\|\sigma(s)\|_{L_{b}^{2}(\mathbb{R} ; H)}^{2} \\
\leq & \left\|u_{\tau}\right\|^{2} e^{-\left(2 \lambda_{1} v+\alpha\right)(t-\tau)}+\frac{4}{\alpha} \frac{1}{\left(1-e^{-\left(2 \lambda_{1} v+\alpha\right)}\right)}\|\sigma(s)\|_{L_{b}^{2}(\mathbb{R} ; H)}^{2} \\
\leq & \left\|u_{\tau}\right\|^{2} e^{-\left(2 \lambda_{1} v+\alpha\right)(t-\tau)}+\frac{4}{\alpha}\left(1+\frac{1}{\left(2 \lambda_{1} v+\alpha\right)}\right)\|\sigma(s)\|_{L_{b}^{2}(\mathbb{R} ; H)}^{2} \\
\leq & \left\|u_{\tau}\right\|^{2} e^{-\left(2 \lambda_{1} v+\alpha\right)(t-\tau)}+\frac{4}{\alpha}\left(1+\frac{1}{\left(2 \lambda_{1} v+\alpha\right)}\right)\left\|\sigma_{0}\right\|_{L_{b}^{2}(\mathbb{R} ; H)}^{2} .
\end{aligned}
$$

Now choosing $\left\|u_{\tau}\right\|^{2} e^{-\left(2 \lambda_{1} v+\alpha\right)(t-\tau)} \leq \frac{4}{\alpha}\left(1+\frac{1}{\left(2 \lambda_{1} v+\alpha\right)}\right)\left\|\sigma_{0}\right\|_{L_{b}^{2}(\mathbb{R} ; H)}^{2}$, there exists a time $t_{0}=$ $t_{0}\left(\alpha, \lambda_{1},\left\|\sigma_{0}\right\|_{L_{b}^{2}(R ; H)}^{2}\right)>0$ such that $B_{0}=\left\{u:\|u\|^{2} \leq \rho^{2}\right\}$, where $\rho^{2}=\frac{8}{\alpha}\left(1+\frac{1}{\left(2 \lambda_{1} v+\alpha\right)}\right) \times$ $\left\|\sigma_{0}\right\|_{L_{b}^{2}(\mathbb{R} ; H)}^{2}$, that is, $B_{0}$ is a uniformly (w.r.t. $\sigma \in \Sigma$ ) absorbing ball for the process $\left\{U_{\sigma}(t, \tau)\right\}$ in $H$.

Lemma 5.2 Let $\left\{u_{\tau}^{n}\right\}$ be a sequence converging weakly to some $u_{\tau}$ in Banach spaces $V, H$, and $\left(L^{4}(\Omega)\right)^{3}$, and $\left\{\sigma^{n}\right\} \in \mathcal{H}\left(\sigma_{0}\right)$ be a sequence converging weakly to some $\sigma \in \mathcal{H}\left(\sigma_{0}\right)$. Then for any fixed $t \geq \tau \in \mathbb{R}, U_{\sigma^{n}}(t, \tau) u_{\tau}^{n} \rightarrow U_{\sigma}(t, \tau) u_{\tau}$ weakly in $L^{2}(\tau, T ; V), C(\tau,+\infty ; H)$, and $L^{4}\left(\tau, T ;\left(L^{4}(\Omega)\right)^{3}\right)$ for all $t \geq \tau$, respectively.

Proof Noting that $\left\{U_{\sigma^{n}}(t, \tau) u_{\tau}^{n}\right\}=\left\{u^{n}(t)\right\}, U_{\sigma}(t, \tau) u_{\tau}=u(t)$, by Theorem 2.1 we know that $\left\{u^{n}(t)\right\}$ and $u$ are bounded in $L^{2}(\tau, T ; V), C(\tau,+\infty ; H)$, and $L^{4}\left(\tau, T ;\left(L^{4}(\Omega)\right)^{3}\right)$, and hence the weak convergence holds.

Lemma 5.3 For any $\sigma \in \Sigma=\mathcal{H}\left(\sigma_{0}\right)$, the family of processes $\left\{U_{\sigma}(t, \tau)\right\}, t \geq \tau \in \mathbb{R}$, generated by the global solution of (2.5)-(2.8) are $(H \times \Sigma, H)$ continuous.

Proof Let $t$ and $\tau$ be fixed, and for any $T>0, t \geq \tau, t, \tau \in[0, T]$, let $\left\{\left(u_{\tau}^{n}, \sigma^{n}\right)\right\} \subset H \times \mathcal{H}\left(\sigma_{0}\right)$ be a sequence that converges to some $\left\{\left(u_{\tau}, \sigma\right)\right\} \subset H \times \mathcal{H}\left(\sigma_{0}\right)$, and let $u^{n}(t)$ and $u(t)$ be the solutions of problem (2.5)-(2.8) with symbols $\sigma^{n}, \sigma$ and initial data $u_{\tau}^{n}, u_{\tau}$, respectively.

Setting

$$
w^{n}(t)=u(t)-u^{n}(t)=U_{\sigma}(t, \tau) u_{\tau}-U_{\sigma^{n}}(t, \tau) u_{\tau}^{n}, \quad n=1,2, \ldots
$$

we can see that $w^{n}(t)$ is a solution of the following problem for each integer $n$ :

$$
\begin{aligned}
& \frac{\partial w^{n}}{\partial t}+v A w^{n}+\alpha w^{n}+B(u)-B\left(u^{n}\right)=\sigma-\sigma^{n}, \\
& \left.w^{n}\right|_{t=\tau}=w_{\tau}^{n}=u_{\tau}-u_{\tau}^{n}, \quad \tau \geq 0 .
\end{aligned}
$$


Taking the inner product of (5.6) with $w^{n}$ in $H$, we get

$$
\begin{aligned}
& \frac{1}{2} \frac{d}{d t}\left(w^{n}, w^{n}\right)+v\left(\nabla w^{n}, \nabla w^{n}\right)+\alpha\left(w^{n}, w^{n}\right)+\left(B(u)-B\left(u^{n}\right), w^{n}\right) \\
& \quad=\left(\sigma-\sigma^{n}, w^{n}\right) \leq \alpha\left(w^{n}, w^{n}\right)+\frac{1}{\alpha}\left\|\sigma-\sigma^{n}\right\|^{2},
\end{aligned}
$$

where

$$
\begin{aligned}
\left|\left(B(u)-B\left(u^{n}\right), w^{n}\right)\right| \\
\leq C\left|\left(\beta|u| u+\gamma|u|^{2} u-\beta\left|u^{n}\right| u^{n}-\gamma\left|u^{n}\right|^{2} u^{n}, w^{n}\right)\right| \\
\leq C \mid\left(\beta|u| u-\beta\left|u^{n}\right| u+\beta\left|u^{n}\right| u-\beta\left|u^{n}\right| u^{n}, w^{n}\right) \\
\quad+\left(\gamma|u|^{2} u-\gamma\left|u^{n}\right|^{2} u+\gamma\left|u^{n}\right|^{2} u-\gamma\left|u^{n}\right|^{2} u^{n}, w^{n}\right) \mid \\
\leq C\left[\beta\left(\|u\|_{V}+\left\|u^{n}\right\|_{V}\right)\right. \\
\left.\quad+\gamma\left(\|u\|_{V}\left\|u_{n}\right\|_{V}+\|u\|_{\left(L^{4}(\Omega)\right)^{3}}+\left\|u^{n}\right\|_{\left(L^{4}(\Omega)\right)^{3}}\right)\right]\left\|w^{n}\right\|^{2} .
\end{aligned}
$$

Hence, from (5.7)-(5.8) it follows that

$$
\begin{aligned}
\frac{1}{2} \frac{d}{d t}\left\|w^{n}(t)\right\|^{2}= & -\left(B(u)-B\left(u^{n}\right), w^{n}\right)+\left(\sigma-\sigma^{n}, w^{n}\right) \\
\leq & \frac{1}{\alpha}\left\|\sigma-\sigma^{n}\right\|^{2}+C\left[\beta\left(\|u\|_{V}+\left\|u^{n}\right\|_{V}\right)\right. \\
& \left.+\gamma\left(\|u\|_{V}\left\|u_{n}\right\|_{V}+\|u\|_{\left(L^{4}(\Omega)\right)^{3}}+\left\|u^{n}\right\|_{\left(L^{4}(\Omega)\right)^{3}}\right)\right]\left\|w^{n}\right\|^{2} .
\end{aligned}
$$

Applying the Gronwall inequality to (5.9), we obtain

$$
\begin{aligned}
\left\|w^{n}(t)\right\|^{2}= & \left\|U_{\sigma}(t, \tau) u_{\tau}-U_{\sigma^{n}}(t, \tau) u_{\tau}^{n}\right\|^{2} \\
\leq & {\left[\left\|u_{\tau}-u_{\tau}^{n}\right\|^{2}+\frac{1}{\alpha} \int_{\tau}^{t}\left\|\sigma(s)-\sigma^{n}(s)\right\|^{2} d s\right] \exp \left\{C \int _ { \tau } ^ { t } \left[\beta\left(\|u\|_{V}+\left\|u^{n}\right\|_{V}\right)\right.\right.} \\
& \left.\left.+\gamma\left(\|u\|_{V}\left\|u_{n}\right\|_{V}+\|u\|_{\left(L^{4}(\Omega)\right)^{3}}+\left\|u^{n}\right\|_{\left(L^{4}(\Omega)\right)^{3}}\right)\right] d s\right\} \\
\leq & {\left[\left\|u_{\tau}-u_{\tau}^{n}\right\|^{2}+\frac{1}{\alpha} \int_{\tau}^{T}\left\|\sigma(s)-\sigma^{n}(s)\right\|^{2} d s\right] } \\
& \times \exp \left\{C \int _ { \tau } ^ { T } \left[\beta\left(\|u\|_{V}+\left\|u^{n}\right\|_{V}\right)\right.\right. \\
& \left.\left.+\gamma\left(\|u\|_{V}\left\|u_{n}\right\|_{V}+\|u\|_{\left(L^{4}(\Omega)\right)^{3}}+\left\|u^{n}\right\|_{\left(L^{4}(\Omega)\right)^{3}}\right)\right] d s\right\} \\
\rightarrow & 0
\end{aligned}
$$

since by Theorem 2.1 the following estimates hold for any fixed $t \geq \tau \in \mathbb{R}$ :

$$
\begin{aligned}
& \exp \left\{C \int_{\tau}^{T}\left[\beta\left(\|u\|_{V}+\left\|u^{n}\right\|_{V}\right)+\gamma\left(\|u\|_{V}\left\|u_{n}\right\|_{V}+\|u\|_{\left(L^{4}(\Omega)\right)^{3}}+\left\|u^{n}\right\|_{\left(L^{4}(\Omega)\right)^{3}}\right)\right] d s\right\} \\
& \quad \leq \exp \left[C(T, \gamma, \beta) \int_{\tau}^{T}\left(\|u\|_{V}^{2}+\left\|u^{n}\right\|_{V}^{2}+\|u\|_{\left(L^{4}(\Omega)\right)^{3}}^{4}+\left\|u^{n}\right\|_{\left(L^{4}(\Omega)\right)^{3}}^{4}\right) d s\right]<+\infty .
\end{aligned}
$$


Therefore, (5.10) implies the $\left(H \times \mathcal{H}\left(\sigma_{0}\right), H\right)$ continuity of the processes $\left\{U_{\sigma}(t, \tau), t \geq\right.$ $\tau \in \mathbb{R}\}, \sigma \in \mathcal{H}\left(\sigma_{0}\right)$, defined on $H$.

Lemma 5.4 Let $\left\{u_{\tau}^{n}\right\}$ be a sequence in $H$ converging strongly to some $u_{\tau} \in H$ in the norm of $H$, and let $\left\{\sigma^{n}\right\}$ be a sequence of $\mathcal{H}\left(\sigma_{0}\right)$ converging strongly to some $\sigma \in \mathcal{H}\left(\sigma_{0}\right)$. Then for any fixed $\tau \in \mathbb{R}, U_{\sigma^{n}}(\cdot, \tau) u_{\tau}^{n} \rightarrow U_{\sigma}(\cdot, \tau) u_{\tau}$ strongly in $L^{2}(\tau, T ; H)$ foe all $t \geq \tau$.

Proof From (5.10) we can derive that for any $t \geq \tau, t, \tau \in[0, T]$, the following inequality holds:

$$
\begin{aligned}
\left\|u(t)-u^{n}(t)\right\|^{2} & \leq\left\|w^{n}(t)\right\|^{2} \\
& =\left\|U_{\sigma}(t, \tau) u_{\tau}-U_{\sigma^{n}}(t, \tau) u_{\tau}^{n}\right\|^{2} \\
& \leq C(T)\left[\left\|u_{\tau}-u_{\tau}^{n}\right\|^{2}+C \int_{\tau}^{t}\left\|\sigma(s)-\sigma^{n}(s)\right\|^{2} d s\right] .
\end{aligned}
$$

Integrating (5.12) over $[\tau, T]$ with respect to $t$, we have

$$
\begin{aligned}
& \int_{\tau}^{T}\left\|u(t)-u^{n}(t)\right\|^{2} d t \\
& \quad \leq \int_{\tau}^{T}\left\|U_{\sigma}(t, \tau) u_{\tau}-U_{\sigma^{n}}(t, \tau) u_{\tau}^{n}\right\|^{2} d t \\
& \quad \leq C \int_{\tau}^{T}\left[\left\|u_{\tau}-u_{\tau}^{n}\right\|^{2}+C \int_{\tau}^{t}\left\|\sigma(s)-\sigma^{n}(s)\right\|^{2} d s\right] d t \\
& \quad \leq C(T) \varepsilon .
\end{aligned}
$$

The proof of the lemma is thus complete.

Lemma 5.5 For any $\sigma \in \mathcal{H}\left(\sigma_{0}\right)$ and $u_{\tau} \in H$, the family of processes $\left\{U_{\sigma}(t, \tau), t \geq \tau \in \mathbb{R}\right\}$, $\sigma \in \mathcal{H}\left(\sigma_{0}\right)$, defined on $H$, corresponding to equations (2.1)-(2.4) is uniformly (w.r.t. $\sigma \in \Sigma$ ) asymptotically compact in $H$.

Proof Assume that $\left\{u_{\tau}^{n}\right\}$ is a bounded sequence in $H,\left\{\sigma^{n}\right\} \subset \mathcal{H}\left(\sigma_{0}\right)$ and $\left\{t_{n}\right\} \in(\tau,+\infty)$, $t_{n} \rightarrow+\infty$ as $n \rightarrow+\infty$.

From the proof of the existence of uniformly absorbing sets we can see that for any fixed $\tau \in \mathbb{R}$, there exists a time $T_{0}=T_{0}(\rho, \tau)$ depending on the radius $\rho$ of the absorbing ball and $\tau$ such that for all $t_{n} \geq T_{0},\left\{U_{\sigma^{n}}\left(t_{n}, \tau\right) u_{\tau}^{n}\right\} \subseteq B_{0}$, where $B_{0}$ is defined in Lemma 5.1.

By Lemma 5.2 the sequence $\left\{U_{\sigma^{n}}\left(t_{n}, \tau\right) u_{\tau}^{n}\right\}$ is weakly precompact in $H$, and hence we have

$$
U_{\sigma^{n}}\left(t_{n}, \tau\right) u_{\tau}^{n} \rightarrow u \quad \text { weakly in } H \text { as } n \rightarrow+\infty
$$

for some $u \in H$ and some subsequence (still denoted by) $U_{\sigma^{n}}\left(t_{n}, \tau\right) u_{\tau}^{n}$.

Similarly, for each $T>0$ and $t_{n} \geq T_{0}+T$,

$$
u_{T}^{n} \doteq U_{\sigma^{n}}\left(t_{n}-T, \tau\right) u_{\tau}^{n} \rightarrow u_{T} \quad \text { weakly in } H \text { as } n \rightarrow+\infty
$$

for some $u_{T} \in H$. 
Noting that the translation semigroup $\{S(h): h \geq 0\}$ satisfies

$$
U_{S(h) \sigma}(t, \tau)=U_{\sigma}(t+h, \tau+h), \quad \forall h \geq 0, t \geq \tau \in \mathbb{R}, \sigma \in \mathcal{H}\left(\sigma_{0}\right),
$$

we see that

$$
\begin{aligned}
U_{\sigma^{n}}\left(t_{n}, \tau\right) & =U_{\sigma^{n}}\left(t_{n}, t_{n}-T\right) U_{\sigma^{n}}\left(t_{n}-T, \tau\right) \\
& =U_{S\left(t_{n}-T\right) \sigma^{n}}(T, 0) U_{\sigma^{n}}\left(t_{n}-T, \tau\right), \quad t_{n}-T \geq \tau .
\end{aligned}
$$

Letting $\sigma_{T}^{n}=S\left(t_{n}-T\right) \sigma^{n}$, from (5.17) and (5.16) we derive

$$
\begin{aligned}
U_{\sigma^{n}}\left(t_{n}, \tau\right) u_{\tau}^{n} & =U_{\sigma_{T}^{n}}(T, 0) U_{\sigma^{n}}\left(t_{n}-T, \tau\right) u_{\tau}^{n} \\
& =U_{\sigma_{T}^{n}}(T, 0) u_{T}^{n}, \quad t_{n}-T \geq \tau .
\end{aligned}
$$

Since $\left\{\sigma_{T}^{n}\right\} \subset \mathcal{H}\left(\sigma_{0}\right)$ and $\Sigma=\mathcal{H}\left(\sigma_{0}\right)$ is compact in $L_{\text {loc }}^{2}(\mathbb{R} ; H)$, there exist a subsequence of $\left\{\sigma_{T}^{n}\right\}$ (also denoted by $\left\{\sigma_{T}^{n}\right\}$ ) and some $\sigma_{T} \in \mathcal{H}\left(\sigma_{0}\right)$ such that

$$
\sigma_{T}^{n} \rightarrow \sigma_{T} \quad \text { strongly in } L_{\mathrm{loc}}^{2}(\mathbb{R} ; H) \text { as } n \rightarrow+\infty, \forall T>0
$$

By Lemmas 5.2 and 5.4 and by formulas (5.14)-(5.15) and (5.18) we conclude

$$
u=U_{\sigma_{T}}(T, 0) u_{T}, \quad \forall T>0
$$

Next, we want to prove the asymptotic compactness in $H$, that is,

$$
\left\|U_{\sigma_{T}^{n}}\left(t_{n}, \tau\right) u_{\tau}^{n}-u\right\| \rightarrow 0 \quad \text { as } n \rightarrow+\infty
$$

that is, it suffices to prove that

$$
\left\|U_{\sigma_{T}^{n}}\left(t_{n}, \tau\right) u_{\tau}^{n}\right\| \rightarrow\|u\| \quad \text { as } n \rightarrow+\infty
$$

and

$$
U_{\sigma_{T}^{n}}\left(t_{n}, \tau\right) u_{\tau}^{n} \rightarrow u \quad \text { in } H \text { as } n \rightarrow+\infty
$$

However, (5.14) clearly ensures (5.23). Next, we only need to prove (5.22).

To this end, we shall prove that

$$
\begin{aligned}
& \liminf _{n \rightarrow+\infty}\left\|U_{\sigma^{n}}\left(t_{n}, \tau\right) u_{\tau}^{n}\right\|=\liminf _{n \rightarrow+\infty}\left\|U_{\sigma_{T}^{n}}(T, 0) u_{T}^{n}\right\| \geq\|u\|, \\
& \limsup _{n \rightarrow+\infty}\left\|U_{\sigma^{n}}\left(t_{n}, \tau\right) u_{\tau}^{n}\right\|=\limsup _{n \rightarrow+\infty}\left\|U_{\sigma_{T}^{n}}(T, 0) u_{T}^{n}\right\| \leq\|u\| .
\end{aligned}
$$

However, the weak convergence of the corresponding sequences (i.e., (5.14) and (5.15)) and Lemma 5.2 ensure that (5.24) is true, so our aim is only to prove (5.25). 
Taking the inner product with the both sides of equation (2.5) by $u$, we obtain

$$
\frac{1}{2} \frac{d}{d t}(u, u)+\alpha(u, u)=(-B(u)-v A u+\sigma, u)=(\sigma(t), u)-G(u(t)),
$$

where $G(u(t))=(B(u)+v A u+\alpha u, u)=(P F(u)+v A u+\alpha u, u)=\left(P\left(\beta|u| u+\gamma|u|^{2} u\right)+v A u+\right.$ $\alpha u, u)$.

Integrating (5.26) over $[\tau, t]$ with respect to the time variable, we derive

$$
\begin{aligned}
\|u\|^{2}= & (u(t), u(t))=(u(\tau), u(\tau)) e^{-2 \alpha(t-\tau)}+2 \int_{\tau}^{t} e^{-2 \alpha(t-s)}(\sigma(s), u(s)) d s \\
& -2 \int_{\tau}^{t} e^{-2 \alpha(t-s)} G(u(s)) d s .
\end{aligned}
$$

Substituting $u(t)$ in (5.27) by $U_{\sigma_{T}^{n}}(T, 0) u_{T}^{n}$ and changing the integration domain to $[0, T]$, we deduce

$$
\begin{aligned}
\left\|U_{\sigma_{T}^{n}}(T, 0) u_{T}^{n}\right\|^{2}= & \left(U_{\sigma_{T}^{n}}(T, 0) u_{T}^{n}, U_{\sigma_{T}^{n}}(T, 0) u_{T}^{n}\right) \\
= & \left(u_{T}^{n}, u_{T}^{n}\right) e^{-2 \alpha T}+2 \int_{0}^{T} e^{-2 \alpha(T-s)}\left(\sigma_{s}^{n}(s), U_{\sigma_{s}^{n}}(s, 0) u_{s}^{n}\right) d s \\
& -2 \int_{0}^{T} e^{-2 \alpha(T-s)} G\left(U_{\sigma_{s}^{n}}(s, 0) u_{s}^{n}\right) d s \\
= & I_{1}+I_{2}+I_{3} .
\end{aligned}
$$

Similarly, substituting $u(t)$ in (5.27) by $U_{\sigma_{T}}(T, 0) u_{T}$ and changing the integration domain to $[0, T]$, we have

$$
\begin{aligned}
\left\|U_{\sigma_{T}}(T, 0) u_{T}\right\|^{2}= & \left(U_{\sigma_{T}}(T, 0) u_{T}, U_{\sigma_{T}}(T, 0) u_{T}\right) \\
= & \left(u_{T}, u_{T}\right) e^{-2 \alpha T}+2 \int_{0}^{T} e^{-2 \alpha(T-s)}\left(\sigma_{s}(s), U_{\sigma_{s}}(s, 0) u_{s}\right) d s \\
& -2 \int_{0}^{T} e^{-2 \alpha(T-s)} G\left(U_{\sigma_{s}}(s, 0) u_{s}\right) d s
\end{aligned}
$$

Next, we shall deal with the right-hand side of (5.28) term by term. From Lemma 5.1 we derive

$$
\underset{n}{\limsup } I_{1}=\limsup _{n}\left(u_{T}^{n}, u_{T}^{n}\right) e^{-2 \alpha T} \leq \rho^{2} e^{-2 \alpha T} .
$$

Since $L^{2}([\tau, T] ; V) \hookrightarrow \hookrightarrow L^{2}([\tau, T] ; H)$, from (5.15), (5.18), and Lemma 5.2 we deduce that

$$
U_{\sigma_{s}^{n}}(s, 0) u_{s}^{n} \rightarrow U_{\sigma_{s}}(s, 0) u_{s} \quad \text { weakly in } L^{2}([\tau, T] ; H), \forall T>\tau
$$

whence we can deduce from (5.31) that

$$
\begin{aligned}
\lim _{n \rightarrow \infty} I_{2} & =\lim _{n \rightarrow \infty} 2 \int_{0}^{T} e^{-2 \alpha(T-s)}\left(\sigma_{s}^{n}(s), U_{\sigma_{s}^{n}}(s, 0) u_{s}^{n}\right) d s \\
& =2 \int_{0}^{T} e^{-2 \alpha^{2}(T-s)}\left(\sigma_{s}(s), U_{\sigma_{s}}(s, 0) u_{s}\right) d s
\end{aligned}
$$


Next, we will deal with $I_{3}$ on the right-hand side of (5.26), which we shall prove in Lemma 5.6 (to be proved later on), that is, we want to prove

$$
\begin{aligned}
\limsup _{n} I_{3} & =\limsup _{n}\left(-2 \int_{0}^{T} e^{-2 \alpha(t-s)} G\left(U_{\sigma_{s}^{n}}(s, 0) u_{s}^{n}\right) d s\right) \\
& =-\liminf _{n}\left(2 \int_{0}^{T} e^{-2 \alpha(t-s)} G\left(U_{\sigma_{s}^{n}}(s, 0) u_{s}^{n}\right) d s\right) \\
& \leq-2 \int_{0}^{T} e^{-2 \alpha(t-s)} G\left(U_{\sigma_{s}}(s, 0) u_{s}\right) d s .
\end{aligned}
$$

Combining (5.29), (5.30), and (5.32)-(5.33), we obtain

$$
\begin{aligned}
\underset{n}{\limsup }\left(\left\|U_{\sigma_{T}^{n}}(T, 0) u_{T}^{n}\right\|^{2}\right)= & \left(U_{\sigma_{T}^{n}}(T, 0) u_{T}^{n}, U_{\sigma_{T}^{n}}(T, 0) u_{T}^{n}\right) \\
\leq & \rho^{2} e^{-2 \alpha T}+2 \int_{0}^{T} e^{-2 \alpha^{2}(T-s)}\left(\sigma_{s}(s), U_{\sigma_{s}}(s, 0) u_{s}\right) d s \\
& -2 \int_{0}^{T} e^{-2 \alpha(t-s)} G\left(U_{\sigma_{s}}(s, 0) u_{s}\right) d s \\
= & \left\|U_{\sigma_{T}}(T, 0) u_{T}\right\|^{2}-\left(u_{T}, u_{T}\right) e^{-2 \alpha T}+\rho^{2} e^{-2 \alpha T} \\
\leq & \left\|U_{\sigma_{T}}(T, 0) u_{T}\right\|^{2}+2 \rho^{2} e^{-2 \alpha T} .
\end{aligned}
$$

Hence, letting $T$ tend to $+\infty$ in (5.34), we derive

$$
\begin{aligned}
\lim _{n \rightarrow \infty}\left\|U_{\sigma^{n}}\left(t_{n}, \tau\right) u_{\tau}^{n}\right\|^{2} & =\limsup _{n, T \rightarrow \infty}\left\|U_{\sigma_{T}^{n}}(T, 0) u_{T}^{n}\right\|^{2} \\
& \leq \limsup _{n, T \rightarrow \infty}\left(\left\|U_{\sigma_{T}}(T, 0) u_{T}\right\|^{2}+2 \rho^{2} e^{-2 \alpha T}\right) \\
& =\left\|U_{\sigma}(t, \tau) u_{\tau}\right\|^{2}=\|u\|^{2},
\end{aligned}
$$

which, along with (5.24), gives

$$
\limsup _{n, T \rightarrow \infty}\left\|U_{\sigma_{T}^{n}}(T, 0) u_{T}^{n}\right\|^{2}=\lim _{n \rightarrow \infty}\left\|U_{\sigma^{n}}\left(t_{n}, \tau\right) u_{\tau}^{n}\right\|^{2}=\left\|U_{\sigma}(t, \tau) u_{\tau}\right\|^{2}=\|u\|^{2} .
$$

Combining the norm convergence (5.36) and weak convergence (5.23), we obtain

$$
\lim _{n \rightarrow \infty}\left\|U_{\sigma_{T}^{n}}\left(t_{n}, \tau\right) u_{\tau}^{n}-u\right\|=0 .
$$

To complete the proof of Lemma 5.5, we need to prove the following lemma.

\section{Lemma 5.6}

$$
\begin{aligned}
\limsup _{n} I_{3} & =\limsup _{n}\left(-2 \int_{0}^{T} e^{-2 \alpha(t-s)} G\left(U_{\sigma_{s}^{n}}(s, 0) u_{s}^{n}\right) d s\right) \\
& =-\liminf _{n}\left(2 \int_{0}^{T} e^{-2 \alpha(t-s)} G\left(U_{\sigma_{s}^{n}}(s, 0) u_{s}^{n}\right) d s\right) \\
& \leq-2 \int_{0}^{T} e^{-2 \alpha(t-s)} G\left(U_{\sigma_{s}}(s, 0) u_{s}\right) d s .
\end{aligned}
$$


Proof From (5.15), (5.18), and Lemma 5.2, by the embedding theorem we have:

$$
\begin{array}{ll}
U_{\sigma_{T}^{n}}(s, 0) u_{T}^{n} \rightarrow U_{\sigma_{T}}(s, 0) u_{T} & \text { weakly in } C(0, T ; H), \\
U_{\sigma_{T}^{n}}(s, 0) u_{T}^{n} \rightarrow U_{\sigma_{T}}(s, 0) u_{T} & \text { strongly in } L^{2}(0, T ; H), \\
U_{\sigma_{T}^{n}}(s, 0) u_{T}^{n} \rightarrow U_{\sigma_{T}}(s, 0) u_{T} & \text { weakly in } L^{4}\left(0, T ;\left(L^{4}(\Omega)\right)^{3}\right) .
\end{array}
$$

To end the proof of (5.38), we should complete the proof of the following formula:

$$
\liminf _{n}\left(\int_{0}^{T} e^{-2 \alpha(t-s)} G\left(U_{\sigma_{s}^{n}}(s, 0) u_{s}^{n}\right) d s\right) \geq \int_{0}^{T} e^{-2 \alpha(t-s)} G\left(U_{\sigma_{s}}(s, 0) u_{s}\right) d s,
$$

where $G(u(t))=(B(u)+v A u, u)=\left(P\left(\beta|u| u+\gamma|u|^{2} u\right)+v A u, u\right)$.

Since $A: D(A) \mapsto H$ is a linear positive definite operator, from (5.39)-(5.41) it follows that

$$
\begin{gathered}
\underset{n}{\liminf } \int_{0}^{T} e^{-2 \alpha(t-s)}\left(v A U_{\sigma_{s}^{n}}(s, 0) u_{s}^{n}, U_{\sigma_{s}^{n}}(s, 0) u_{s}^{n}\right) d s \\
\geq \int_{0}^{T} e^{-2 \alpha(t-s)}\left(v A U_{\sigma_{s}}(s, 0) u_{s}, U_{\sigma_{s}}(s, 0) u_{s}\right) d s .
\end{gathered}
$$

Next, we want to prove that

$$
\begin{gathered}
\lim _{n \rightarrow+\infty} \int_{0}^{T} e^{-2 \alpha(t-s)}\left(B\left(U_{\sigma_{s}^{n}}(s, 0) u_{s}^{n}\right), U_{\sigma_{s}^{n}}(s, 0) u_{s}^{n}\right) d s \\
=\int_{0}^{T} e^{-2 \alpha(t-s)}\left(B\left(U_{\sigma_{s}}(s, 0) u_{s}\right), U_{\sigma_{s}}(s, 0) u_{s}\right) d s .
\end{gathered}
$$

Setting

$$
\begin{aligned}
& K_{1}^{n}(s)=\left(B\left(U_{\sigma_{s}^{n}}(s, 0) u_{s}^{n}\right), U_{\sigma_{s}^{n}}(s, 0) u_{s}^{n}\right), \\
& K_{2}(s)=\left(B\left(U_{\sigma_{s}}(s, 0) u_{s}\right), U_{\sigma_{s}}(s, 0) u_{s}\right),
\end{aligned}
$$

we consider

$$
\begin{aligned}
I= & \left|\int_{0}^{T} e^{-2 \alpha(t-s)}\left(K_{2}^{n}(s)-K_{1}^{n}(s)\right) d s\right| \\
\leq & \mid \int_{0}^{T} e^{-2 \alpha(t-s)}\left(\beta\left|U_{\sigma_{s}}(s, 0) u_{s}\right| U_{\sigma_{s}}(s, 0) u_{s}+\gamma\left|U_{\sigma_{s}}(s, 0) u_{s}\right|^{2} U_{\sigma_{s}}(s, 0) u_{s}\right. \\
& -\beta\left|U_{\sigma_{s}^{n}}(s, 0) u_{s}^{n}\right| U_{\sigma_{s}^{n}}(s, 0) u_{s}^{n}-\gamma\left|U_{\sigma_{s}^{n}}(s, 0) u_{s}^{n}\right|^{2} U_{\sigma_{s}^{n}}(s, 0) u_{s}^{n} \\
& \left.U_{\sigma_{s}}(s, 0) u_{s}-U_{\sigma_{s}^{n}}(s, 0) u_{s}^{n}\right) d s \mid \\
\leq & C \mid \int_{0}^{T} e^{-2 \alpha(t-s)}\left(\beta\left|U_{\sigma_{s}}(s, 0) u_{s}\right| U_{\sigma_{s}}(s, 0) u_{s}-\beta\left|U_{\sigma_{s}^{n}}(s, 0) u_{s}^{n}\right| U_{\sigma_{s}}(s, 0) u_{s}\right. \\
& +\beta\left|U_{\sigma_{s}^{n}}(s, 0) u_{s}^{n}\right| U_{\sigma_{s}}(s, 0) u_{s}-\beta\left|U_{\sigma_{s}^{n}}(s, 0) u_{s}^{n}\right| U_{\sigma_{s}^{n}}(s, 0) u_{s}^{n} \\
& \left.U_{\sigma_{s}}(s, 0) u_{s}-U_{\sigma_{s}^{n}}(s, 0) u_{s}^{n}\right)
\end{aligned}
$$




$$
\begin{aligned}
& +\left(\gamma\left|U_{\sigma_{s}}(s, 0) u_{s}\right|^{2} U_{\sigma_{s}}(s, 0) u_{s}-\gamma\left|U_{\sigma_{s}^{n}}(s, 0) u_{s}^{n}\right|^{2} U_{\sigma_{s}}(s, 0) u_{s}\right. \\
& +\gamma\left|U_{\sigma_{s}^{n}}(s, 0) u_{s}^{n}\right|^{2} U_{\sigma_{s}}(s, 0) u_{s}-\gamma\left|U_{\sigma_{s}^{n}}(s, 0) u_{s}^{n}\right|^{2} U_{\sigma_{s}^{n}}(s, 0) u_{s}^{n}, \\
& \left.U_{\sigma_{s}}(s, 0) u_{s}-U_{\sigma_{s}^{n}}(s, 0) u_{s}^{n}\right) d s \mid \\
\leq & C \mid \int_{0}^{T} e^{-2 \alpha(t-s)}\left[\beta\left(\left\|U_{\sigma_{s}}(s, 0) u_{s}\right\|_{V}+\left\|U_{\sigma_{s}^{n}}(s, 0) u_{s}^{n}\right\|_{V}\right)\right. \\
& +\gamma\left(\left\|U_{\sigma_{s}}(s, 0) u_{s}\right\|_{V}\left\|U_{\sigma_{s}^{n}}(s, 0) u_{s}^{n}\right\|_{V}+\left\|U_{\sigma_{s}}(s, 0) u_{s}\right\|_{\left(L^{4}(\Omega)\right)^{3}}\right. \\
& \left.\left.+\left\|U_{\sigma_{s}^{n}}(s, 0) u_{s}^{n}\right\|_{\left(L^{4}(\Omega)\right)^{3}}\right)\right]\left\|U_{\sigma_{s}}(s, 0) u_{s}-U_{\sigma_{s}^{n}}(s, 0) u_{s}^{n}\right\|^{2} d s \mid \\
\leq & C \mid \int_{0}^{T} e^{-4 \alpha(t-s)} \beta\left(\left\|U_{\sigma_{s}}(s, 0) u_{s}\right\|_{V}^{2}+\left\|U_{\sigma_{s}^{n}}(s, 0) u_{s}^{n}\right\|_{V}^{2}\right) \\
& +\gamma\left(\left\|U_{\sigma_{s}}(s, 0) u_{s}\right\|_{V}^{2}\left\|U_{\sigma_{s}^{n}}(s, 0) u_{s}^{n}\right\|_{V}^{2}+\left\|U_{\sigma_{s}}(s, 0) u_{s}\right\|_{\left(L^{4}(\Omega)\right)^{3}}^{4}\right. \\
& \left.+\left\|U_{\sigma_{s}^{n}}(s, 0) u_{s}^{n}\right\|_{\left(L^{4}(\Omega)\right)^{3}}^{4}+C\right) d s \mid \\
& \times\left|\sup _{s \in[0, T]}\left\|U_{\sigma_{s}}(s, 0) u_{s}-U_{\sigma_{s}^{n}}(s, 0) u_{s}^{n}\right\|^{4}\right| .
\end{aligned}
$$

By Theorem 2.1, Lemma 5.2, and Lemma 5.4, since

$$
\begin{aligned}
& \mid \int_{0}^{T} e^{-4 \alpha(t-s)} \beta\left(\left\|U_{\sigma_{s}}(s, 0) u_{s}\right\|_{V}^{2}+\left\|U_{\sigma_{s}^{n}}(s, 0) u_{s}^{n}\right\|_{V}^{2}\right) \\
& \quad+\gamma\left(\left\|U_{\sigma_{s}}(s, 0) u_{s}\right\|_{V}^{2}\left\|U_{\sigma_{s}^{n}}(s, 0) u_{s}^{n}\right\|_{V}^{2}+\left\|U_{\sigma_{s}}(s, 0) u_{s}\right\|_{\left(L^{4}(\Omega)\right)^{3}}^{4}\right. \\
& \left.\quad+\left\|U_{\sigma_{s}^{n}}(s, 0) u_{s}^{n}\right\|_{\left(L^{4}(\Omega)\right)^{3}}^{4}+C\right) d s \mid \\
& \quad<+\infty
\end{aligned}
$$

we conclude

$$
I \leq C(T) \sup _{s \in[0, T]}\left\|U_{\sigma_{s}}(s, 0) u_{s}-U_{\sigma_{s}^{n}}(s, 0) u_{s}^{n}\right\|^{4} \rightarrow 0
$$

as $n \rightarrow \infty$, that is,

$$
\begin{aligned}
\lim _{n \rightarrow+\infty} I= & \lim _{n \rightarrow+\infty} \int_{0}^{T} e^{-2 \alpha(t-s)}\left(K_{2}^{n}(s)-K_{1}^{n}(s)\right) d s \\
= & \lim _{n \rightarrow+\infty} e^{-2 \alpha(t-s)}\left(B\left(U_{\sigma_{s}}(s, 0) u_{s}\right), U_{\sigma_{s}}(s, 0) u_{s}\right) \\
& \left.-\left(B\left(U_{\sigma_{s}^{n}}(s, 0) u_{s}^{n}\right), U_{\sigma_{s}^{n}}(s, 0) u_{s}^{n}\right)\right) d s \\
= & 0 .
\end{aligned}
$$


Remark 5.1 For all bounded sets $B \in H$,

$$
\mathcal{A}_{\Sigma}=\omega_{\tau, \mathcal{H}\left(\sigma_{0}\right)}(B) \doteq \bigcup_{B \in \mathcal{B}(H)} \bigcap_{t \geq \tau} \overline{\bigcup_{\sigma \in \mathcal{H}\left(\sigma_{0}\right)} \bigcup_{s \geq t} U_{\sigma}(s, \tau) B} .
$$

Combining the uniformly (w.r.t. $\sigma \in \mathcal{H}\left(\sigma_{0}\right)$ ) absorbing property (i.e., Lemma 5.1), the continuity of the process (i.e., Lemma 5.3), the asymptotic compactness property of the processes $\left\{U_{\sigma}(t, \tau)\right\}\left(\sigma \in \mathcal{H}\left(\sigma_{0}\right)\right)$ in $H$ (i.e., Lemma 5.5), from Theorem 3.1 we deduce the existence of a uniform attractor in the following theorem.

Theorem 5.1 Assume that $u_{\tau} \in H, \sigma \in \Sigma \subset L_{\text {loc }}^{2}(\mathbb{R} ; H)$. Then the family of processes $\left\{U_{\sigma}(t, \tau), t \geq \tau \in \mathbb{R}\right\}\left(\sigma \in \mathcal{H}\left(\sigma_{0}\right)\right)$ generated by the global solution of problem (1.1) or (2.5)(2.8) possesses a uniform (w.r.t. $\left.\sigma \in \Sigma=\mathcal{H}\left(\sigma_{0}\right)\right)$ attractor $\mathcal{A}_{\mathcal{H}\left(\sigma_{0}\right)}=\mathcal{A}_{\Sigma}$ in $H$.

Competing interests

The authors declare that they have no competing interests.

\section{Authors' contributions}

The authors declare that the work was realized in collaboration with the same responsibility. All authors read and approved the final manuscript.

\section{Author details}

${ }^{1}$ College of Education and Teacher Development, Henan Normal University, Xinxiang, 453007, P.R. China. ${ }^{2}$ Information and Management Science, Henan Agricultural University, Zhengzhou, 450046, P.R. China. ${ }^{3}$ College of Science, Information Engineering University, Zhengzhou, 450001, P.R. China.

\section{Acknowledgements}

This work was in part supported by the Program for Science and Technology Innovation Talents in University of Henan Province (No. 142102210448) and the Mainstay Fund from Henan Normal University. The authors thank the referees by his/her comments, which led to improvements in the presentation of this paper.

Received: 20 November 2015 Accepted: 4 January 2016 Published online: 18 January 2016

\section{References}

1. Gilver, RC, Altobelli, SA: A determination of effective viscosity for the Brinkman-Forchheimer flow model. J. Fluid Mech. 370, 258-355 (1994)

2. Nield, DA: The limitations of the Brinkman-Forchheimer equation in modeling flow in a saturated porous medium and at an interface. Int. J. Heat Fluid Flow 12, 269-272 (1991)

3. Vafai, K, Kim, S: Fluid mechanics of the interface region between a porous medium and a fluid layer - an exact solution. Int. J. Heat Fluid Flow 11(3), 254-256 (1990)

4. Vafai, K, Kim, SJ: On the limitations of the Brinkman-Forchheimer-extended Darcy equation. Int. J. Heat Fluid Flow 16(1), 11-15 (1995)

5. Whitaker, S: The Forchheimer equation: a theoretical development. Transp. Porous Media 25, 27-62 (1996)

6. Uğurlu, D: On the existence of a global attractor for the Brinkman-Forchheimer equations. Nonlinear Anal. TMA 68, 1986-1992 (2008)

7. Ouyang, Y, Yang, L: A note on the existence of a global attractor for the Brinkman-Forchheimer equations. Nonlinear Anal. TMA 70, 2054-2059 (2009)

8. Çelebi, AO, Kalantarov, VK, Uğurlu, D: On continuous dependence on coefficients of the Brinkman-Forchheime equations. Appl. Math. Lett. 19, 801-807 (2006)

9. Celebi, AO, Kalantarov, VK, Uğurlu, D: Continuous dependence for the covective Brinkman-Forchheimer equations. Appl. Anal. 84(9), 877-888 (2005)

10. Liu, Y: Convergence and continuous dependence for the Brinkman-Forchheimer equations. Math. Comput. Model. 49, 1401-1415 (2009)

11. Payne, LE, Song, J, Straughan, B: Continuous dependence and convergence results for Brinkman and Forchheimer models with variable viscosity. Proc. R. Soc. Lond. A 45, 2173-2190 (1999)

12. Wang, B, Lin, S: Existence of global attractors for the three-dimensional Brinkman-Forchheimer equation. Math. Methods Appl. Sci. 31, 1479-1495 (2008)

13. Song, X: Pullback $\mathcal{D}$-attractors for a non-autonomous Brinkman-Forcheimer system. J. Math. Res. Appl. 33(1), 90-100 (2013)

14. Lu, S, Wu, H, Zhong, CK: Attractors for non-autonomous 2D Navier-Stokes equations with normal external forces. Discrete Contin. Dyn. Syst. 13(3), 701-719 (2005)

15. Kang, JR, Park, JY: Uniform attractors for non-autonomous Brinkman-Forchheimer equations with delay. Acta Math. Sin. 29(5), 993-1006 (2013)

16. Kalantarov, VK, Zelik, S: Smooth attractors for the Brinkman-Forchheimer equations with fast growing nonlinearities arXiv:1 101.4070v1 [Math. AP] 21 Jan. (2011) 
17. Zhao, C, You, Y: Approximation of the incompressible convective Brinkman-Forchheimer equations. J. Evol. Equ. 12, 767-788 (2012)

18. Song, $X$, Hou, Y: Uniform attractors for a non-autonomous Brinkman-Forchheimer equation. J. Math. Res. Appl. 32(1), 63-75 (2012)

19. You, Y, Zhao, C, Zhou, S: The existence of uniform attractors for 3D Brinkman-Forchheimer equations. Discrete Contin. Dyn. Syst. 32(10), 3787-3800 (2012)

20. Zelik, S: Asymptotic regularity of solutions of a non-autonomous damped wave equation with a critical growth exponent. Commun. Pure Appl. Anal. 3(4), 921-934 (2004)

21. Chepyzhov, W, Vishik, MI: Attractors for Equations of Mathematical Physics. Am. Math. Soc., Providence (2001)

Submit your manuscript to a SpringerOpen ${ }^{\circ}$ journal and benefit from:

- Convenient online submission

- Rigorous peer review

Immediate publication on acceptance

- Open access: articles freely available online

- High visibility within the field

- Retaining the copyright to your article

Submit your next manuscript at $\boldsymbol{s p r i n g e r o p e n . c o m ~}$ 